\title{
Calendrier, représentations mentales et procédures de calcul chez les Nahuas (Mexique)
}

\section{Marie-Noëlle Chamoux}

\section{(2) OpenEdition \\ 1 Journals}

Édition électronique

URL : https://journals.openedition.org/jsa/3733

DOI : 10.4000/jsa.3733

ISSN : $1957-7842$

Éditeur

Société des américanistes

Édition imprimée

Date de publication : 1 janvier 2003

Pagination : 21-38

ISSN : 0037-9174

\section{Référence électronique}

Marie-Noëlle Chamoux, «Calendrier, représentations mentales et procédures de calcul chez les

Nahuas (Mexique) », Journal de la Société des américanistes [En ligne], 89-1 | 2003, mis en ligne le 16 janvier 2008, consulté le 02 septembre 2022. URL : http://journals.openedition.org/jsa/3733 ; DOI : https://doi.org/10.4000/jsa.3733 


\title{
CALENDRIER, REPRÉSENTATIONS MENTALES ET PROCÉDURES DE CALCUL CHEZ LES NAHUAS (MEXIQUE)
}

\author{
Marie-Noëlle CHAMOUX*
}

\begin{abstract}
Pour le calcul des dates de fêtes importantes se rapportant à la Toussaint ou Fête des Morts, les Nahuas de la région de Huauchinango (Puebla) utilisaient, il y a peu, un système de repères qui s'avère être une mesure exacte, à condition d'abandonner notre préjugé spontané d'une numération séquentielle et de reconstruire des représentations mentales sous-jacentes de type topologique. Le temps des " paysans » n'est pas nécessairement, dans tous les domaines de la vie sociale, ce " temps flottant » dont parlait l'historien Lucien Febvre. [MоTS CLÉS : Nahuatl, calendrier, calcul, représentation mentale, Toussaint, fête des Morts.]
\end{abstract}

Calendar, mental representations and computation procedures among the Nahuas of Huauchinango (Mexico). For determining important dates of feasts related to All Saints' Day or All Souls' Day, the Nahuas of Huauchinango (Puebla) formerly used a system of timemarks which was in fact very precise, so long as it is not judged according to our habits of sequential numeration. Instead, one must reconstruct the topological representations underlying this system. The " peasants' time » is not always this « floating time » defined by Lucien Febvre. [KEY worDS : Nahuatl, calendar, comput, mental representation, All Saints' Day, All Souls' Day.]

Calendario, representaciones mentales y procedimientos de cómputo entre los nahuas (México). Para determinar las fechas de fiestas importantes relacionadas con Todos Santos o Fiesta de los Muertos, los nahuas de la región de Huauchinango (Puebla) empleaban hace poco un sistema de hitos que resulta ser una medición exacta bajo la condición de abandonar nuestro prejuicio espontáneo de una numeración secuencial y de reconstruir representaciones mentales subyacentes de tipo topológico. El tiempo de los " campesinos » no es siempre, en cada ámbito de la vida social, este " tiempo flotante " del cual hablaba el historiador Lucien Febvre. [PALABras CLAves : Náhuatl, calendario, cómputo, representación mental, Todos Santos, Fiesta de los Muertos.]

* Chargée de recherche au CNRS, Centre d'études des langues indigènes d'Amérique, umr 8133, 7 rue Guy Môquet, BP 8, 94801 Villejuif cedex, France [chamoux@vjf.cnrs.fr].

Journal de la Société des Américanistes, 2003,89-1 :p. 21 à 38. Copyright (C) Société des Américanistes. 
Dans la cognition humaine, ce n'est peut-être pas l'idée même de temps en général qui pose le plus de problème - chaque langue l'organise d'une manière ou d'une autre dans son lexique et sa grammaire -, mais la quantification du temps. Cela est connu et a été montré expérimentalement à de nombreuses reprises depuis que Michel Siffre, au début des années soixante, s'est isolé volontairement dans un gouffre sans instruments de mesure temporelle et y a rapidement perdu la possibilité d'évaluer correctement la durée. Contrairement au maniement de la langue maternelle, à l'évaluation de distances et de positions dans un espace familier, à diverses techniques du corps, estimer une durée, se situer soi-même dans le temps ou y localiser un événement devient rarement une habileté mentale et corporelle automatique.

L'existence, dans de nombreuses sociétés humaines, de calendriers, de systèmes de repérage s'appuyant sur l'environnement physique et social, voire sur l'usage d'instruments de mesure élaborés, peut sembler, entre autres fonctions, répondre à cette difficulté cognitive, mais elle ne la résout que partiellement. L'embarras ressenti dans le maniement des unités de temps persiste et ne concerne pas que des populations « archaïques » ou illettrées. Qui d'entre nous n'a jamais hésité sur la date courante, sur le nom du jour? Pourquoi tant d'éphémérides, d'almanachs, d'agendas, de tableaux des jours, etc., dans nos maisons, nos bureaux, nos rues? Quant aux ouvrages d'anthropologie, ils mentionnent si souvent cette gêne qu'il serait fastidieux d'énumérer toutes les observations empiriques faites à ce propos (Evans-Pritchard 1968 ; Bernot et Blancard 1953 ; etc.).

À dire vrai, un calendrier comme système pour rendre discret (au sens des mathématiques) et pour quantifier le temps n'est pas ce qu'il paraît être selon le critère utilitariste naïf du « bon sens ». Il n'est pas l'auxiliaire pratique que l'on croit pour jalonner la durée, la mesurer et situer un événement. Chez l'adulte, il ne se constitue pas en automatisme à l'exception des fragments de langage appris dans l'enfance et mémorisés par cœur : listes des noms des nombres, des unités de temps (jours, mois, etc.). En somme, un calendrier est lui-même un dispositif dans lequel on a besoin de se repérer.

Entre un système de calendrier et son maniement, il existe donc une distance, un vide qui se comble d'événements de toutes sortes. Certains événements peuvent concerner, selon les sociétés, le contrôle de l'ordre social, les rythmes collectifs, le pouvoir; d'autres peuvent renvoyer au savoir, notamment sur le monde, et à ses modalités de partage ; enfin, partout, cette distance s'emplit de procédures, plus ou moins ingénieuses, qui ne sont pas les modes de repérage et de calcul du calendrier officiel, mais des pratiques quotidiennes, du type de celles dont Bruner (1956) préconise l'étude. Ces dernières, bien mal connues encore, font l'objet de notre propos et on a pu en observer des traces chez des Indiens mésoaméricains contemporains.

Un exemple de ces procédures calendaires est une pratique bien connue en France, et peut-être ailleurs. Les adultes, parents ou instituteurs, n'exigent géné- 
ralement pas des écoliers français la mémoire automatique ou « par cœur » du nombre de jours par mois, variable dans le calendrier grégorien actuellement en vigueur ; ils leur transmettent un moyen officieux de repérage à l'aide de leur corps. Sur la main fermée, chacun peut donner le nom d'un mois aux articulations de la base des doigts et aux creux qui les séparent, chaque saillie indiquant un mois « long » de 31 jours et chaque creux un mois « court ». Il suffit d'initialiser le système, en affectant au mois de janvier, premier de la liste, la première bosse au bord de la main et énumérer les mois dans l'ordre du calendrier en les mettant en correspondance avec l'alternance des saillies et des creux.

Dans l'aire méso-américaine contemporaine, la distance entre le calendrier et son usage pratique est particulièrement diffīicile à réduire car le système officiel utilisé par les autorités centrales, État et Église, est le calendrier grégorien qui est importé - il est vrai depuis longtemps.

Chez les anciens Aztèques (de langue nahuatl) et chez les autres peuples précolombiens du Mexique et du Guatemala, le calendrier et la date étaient une véritable obsession puisque chaque moment était sous l'influence d'un dieu ou d'un être " surnaturel »; et les gens croyaient que cela agissait sur les événements. Ce point était si important pour la vie collective et individuelle qu'il existait des spécialistes pour dire et interpréter le calendrier et pour faire des prédictions selon la date. Mais c'était il y a plus de 400 ans et on ne connaît pas très bien l'évolution ultérieure des conceptions et des pratiques liées au calendrier, soumises à la pression coloniale espagnole et à la christianisation. Toujours est-il que je n'ai pas retrouvé de trace manifeste de calendriers précolombiens dans les populations dont je parle ici ${ }^{1}$.

Tout aussi discrète est la présence de survivances susceptibles d'être rattachées à certaines croyances existant dans l'Europe $\mathrm{du} \mathrm{XvI}^{\mathrm{e}}$ siècle, contemporaine de la conquête du Mexique : astrologie, horoscope, kabbale, etc., systèmes qui se fondent également sur des correspondances entre des moments, des astres, des divinités païennes, des anges et des démons.

En revanche, j'ai pu observer les diffïcultés subsistantes que rencontraient des paysans de langue nahuatl (ou aztèque) du Mexique aux environs de Huauchinango (État de Puebla) pour maitriser le calendrier grégorien et certaines procédures qu'ils employaient pour résoudre le problème à une époque - entre 1970 et 1980 - où la plupart d'entre eux étaient analphabètes ou illettrés.

La voie de l'interprétation par les survivances culturelles étant fermée, force est donc de partir des hypothèses sociologiques et anthropologiques sur le temps. Si l'on considère les Nahuas du point de vue de leur situation de paysans, nous disposons d'un modèle classique d'interprétation, celui du « temps flottant ». 


\section{LE MODÈLE CLASSIQUE : TEMPS PAYSAN = TEMPS FLOTTANT}

Sur le thème du temps, l'approche anthropologique dominante se fonde sur une interprétation élaborée au début du $\mathrm{xx}^{\mathrm{e}}$ siècle par l'école française de sociologie (Mauss 1906, 1909 ; Durkheim 1912 ; pour les synthèses récentes en français, voir Izard 1990 ; Bensa 1997). Le temps et son mode local de découpage sont considérés comme des phénomènes collectifs et institutionnels. Leur soubassement se trouverait dans les expériences communes d'un groupe, qui comportent des pics émotionnels correspondant à des moments marquants dans l'environnement naturel, dans les institutions sociales et dans les fêtes. Chez les historiens, cette approche renvoie au célèbre modèle du "temps flottant» proposé par Lucien Febvre pour l'Europe du Xvi ${ }^{\mathrm{e}}$ siècle. Rappelons-en les grandes lignes.

Cet auteur a décrit un type de scansion du temps qu'il nomme «à la paysanne » et où les gens emploient, dit-il, le repérage et non pas la mesure. À l'époque considérée, la précision et l'exactitude n'étaient pas l'objectif. Il y avait très peu d'horloges et elles étaient très imparfaites ${ }^{2}$. Les gens ne savaient pas non plus leur âge exact. Et ce n'était pas seulement vrai pour les illettrés : Erasme ne connaissait pas sa date de naissance mais savait seulement qu'il avait vu le jour la veille de la Saint-Simon et Saint-Jude, repère emprunté à la vie religieuse. Le mois de naissance était rappelé par sa correspondance avec certains travaux agricoles ou des circonstances climatiques ; l'heure était mémorisée par la mère. Mais le millésime de l'année était facilement oublié. À cette époque, le calendrier astronomique n'était pas le système de référence véritable, sauf pour une infime minorité de privilégiés pour qui on tirait l'horoscope (Febvre 1968). Les pratiques temporelles relevées naguère auprès des Nahuas du $\mathrm{xx}^{\mathrm{e}}$ siècle paraissent entrer sans peine dans un tel modèle. Nous pouvons presque nous croire dans l'Europe du XvI ${ }^{\mathrm{e}}$ siècle en ce qui concerne le temps. Bien que le modèle du « temps flottant » ait ses limites, on le verra, il permet, dans une première étape, de camper brièvement le tableau.

Chez les Nahuas, à l'époque de mes observations, la datation était un exercice ni allant de soi ni facile, que ce fût pour évoquer un événement ou pour situer le jour courant. Cela contraste fortement avec les textes anciens en nahuatl qui nous sont parvenus et qui sont émaillés de dates. Ces textes, il est vrai, ont été rédigés, pour la plupart, par des clercs indiens sous la houlette de religieux espagnols et non par des gens du commun (maseualtin). Nous ne connaissons pas ce que savaient vraiment ces derniers, en matière de calendrier.

Dans le village de Cuacuila, vers 1970, le calendrier grégorien était mal maîtrisé. Seuls ceux qui étaient allés à l'école, encore peu nombreux à l'époque, ou qui étaient en contact fréquent pour affaires avec les gens des villes en avaient une bonne connaissance. La plupart des gens-les femmes notamment-ne pouvaient pas donner la date du jour et ignoraient totalement le millésime de l'année en 
cours. Peu savaient vraiment leur âge et leur date de naissance attestée, c'est-àdire pas celle inventée après coup, pour quelque motif administratif.

Pourtant les supports matériels de repérage des dates n'étaient ni rares, ni inaccessibles dans les villages nahuas. Ils étaient sûrement plus répandus que dans l'Europe du Xvi ${ }^{\mathrm{e}}$ siècle. Sur tous les marchés, on pouvait - et on peut toujours acheter un almanach populaire, comme le "Galván ». Mais seules quelques familles nahuas en possédaient et il fallait, de plus, savoir lire pour s'en servir. On le trouvait surtout chez les récitants (rezanderos) qui, lors des enterrements, psalmodient des oraisons catholiques contenues dans des recueils de prières. L'almanach du récitant était consulté à la demande, par un voisin ou un parent, par exemple pour y chercher un prénom pour un nouveau-né. Les calendriers muraux, en revanche, étaient présents dans presque toutes les maisons indiennes, mais beaucoup de gens ne savaient pas les déchiffrer. De fait, c'est peut-être leur illustration - images de Vierge, de saints, d'Aztèque emplumé ou de scènes bucoliques - qui incitait à les afficher.

Pour ce qui est de l'heure on pouvait la qualifier de «flottante». Les gens, en effet, ne cherchaient pas à déterminer l'heure exacte, mais le moment de la journée, au bornage flou, pour régler leurs activités quotidiennes : labeur, repas, repos, etc. Il n'y avait, dans le village, ni horloge, ni cadran solaire, dans aucune maison, au coin d'aucune rue, et pas davantage sur le clocher de l'église. Les seules montres se trouvaient au poignet des instituteurs et de l'anthropologue. Les cloches de l'église ne disaient pas l'heure exacte, mais marquaient le début des activités diurnes : les sacristains - jeunes hommes du village nommés pour un an à cette fonction - étaient chargés de les sonner " à 5 heures du matin 》, disaientils, les lundi, mercredi et dimanche et lors de certaines fètes religieuses. Cette heure est considérée dans le village comme le point du jour, bien que l'obscurité règne encore. Quant à la précision de cet horaire, $\mathrm{j}$ 'ai eu maintes fois l'occasion de vérifier que les activités et les rites prescrits à " 5 heures du matin », selon les Nahuas, pouvaient très bien avoir lieu en réalité à 4 heures et demie, au vu de ma montre. Cependant, si cela s'avérait nécessaire, la plupart possédaient un moyen de repérer des heures en cours de journée, qui n'était ni la hauteur estimée du soleil ni le chant du coq ni un autre phénomène naturel : c'était la radio, allumée du lever au coucher, qui donnait l'heure en espagnol. Les pressions externes pour imposer des horaires rigoureux et minutés étaient encore faibles. Le seul horaire assez précis à respecter était celui de l'école, mais il ne concernait que les familles des enfants effectivement scolarisés (tous les scolarisables ne la fréquentaient pas). Le premier réveil entra par mon intermédiaire, sur demande expresse d'une famille du village. Il accompagna le passage au salariat du père de famille, obligé de respecter une heure précise pour prendre un autocar et aller à son travail d'ouvrier. Son épouse, un jour, me dit que la radio ne suffisait pas et qu'il leur manquait un réveil. Comme elle ne savait où s'en procurer, elle me demanda de lui en apporter un de la ville. 
Les « flottements », on le voit, n'étaient pas éliminés par la simple présence de supports matériels et d'instruments de mesure précis. Il fallait en plus une bonne raison de les réduire : on la trouve non dans un quelconque choix culturel, mais dans les contraintes bien concrètes exercées par les institutions sociales dominantes.

La présentation à la manière de Lucien Febvre laisse de côté tout un ensemble de phénomènes de scansion du temps, peut-être parce qu'ils n'apparaissent pas dans les archives mais, peut-être aussi, parce qu'il ne les a pas cherchés. Il ne se demande pas comment par exemple était repérée la Saint-Simon et Saint-Jude qui servait à Erasme pour situer sa naissance. Or, sur le terrain mexicain, j'ai recueilli un système de calcul pour déterminer le jour de certains Saints.

De fait, l'expression de " temps flottant » est confuse : elle semble renvoyer, d'une part, au principe du repérage et, d'autre part, à un constat d'imprécision, de flou, de vague. Ce n'est pas tout à fait la même chose. Le repérage est un procédé universel qui consiste à décider d'un ou plusieurs jalons et, de ce fait, à effectuer des césures qui " discrétisent » le continu, comme on dit en mathématique ; les mesures de toutes natures appliquent ce procédé, en lui ajoutant une exigence de régularité, mais en conservant, selon les domaines, de fortes variations de finesse des unités découpées : dans le monde contemporain, selon les cas, on utilisera l'unité « jour » ou bien celle de " nanoseconde ». L'imprécision est autre : elle renvoie parfois à l'absence de jalon (le continu), ou alors elle évoque une zone plus ou moins étendue qui fait frontière ou dans laquelle se place parfois un jalon mobile (approximation) ou encore elle suggère une unité de mesure très grossière.

D’une façon générale, l'approche par le " temps flottant » sous-estime ce qui ne flotte pas. Elle ne relève que les absences, les faits en négatif. Elle ignore l'existence de savoirs spécifiquement liés au temps, à son comput et aux procédures officieuses qui l'accompagnent.

\section{UN REPÉRAGE DE FÊTES}

Malgré leur mauvaise maîtrise du calendrier grégorien, tous les Nahuas adultes détenaient des connaissances de base qui lui étaient liées. Ils avaient parfaitement en mémoire les listes ordonnées des noms des mois et des jours en langue espagnole. Ils s'en servaient couramment et de façon correcte. Ils pouvaient dire le nom du jour courant de la semaine (lunes, martes...) et très souvent aussi celui du mois en cours (enero, febrero...).

Ces listes sont insuffisantes pour déterminer une date. Les Indiens ne pouvaient cependant pas en rester là. Sous la colonisation espagnole à l'époque de l'Ancien Régime, le calendrier était réglé par l'Église et les rites catholiques, imposés à tous, du Roi aux Indiens. Ces derniers, pour ne pas être suspectés d'idolâtrie, étaient contraints de se montrer bons chrétiens en suivant les pres- 
criptions rituelles catholiques. De là viennent la plupart des fêtes que nous observons aujourd'hui dans les villages et qui ont été appropriées par les Indiens. Actuellement le calendrier grégorien est celui de l'État moderne et s'impose comme exigence administrative et pratique.

Comment ceux qui n'étaient pas instruits s'y prenaient-ils pour repérer des dates importantes ? La question est loin d'être résolue dans sa globalité et pour tous les domaines de la vie sociale, mais, pour ce qui concerne spécifiquement le cycle des activités rituelles, un système fut décrit spontanément par un informateur de Cuacuila et confirmé par d'autres ${ }^{3}$. Son analyse et la reconstitution de représentations mentales sous-jacentes forment le cœur de cet article.

Voici l'information brute obtenue au sujet de quatre fêtes religieuses :

La Saint-Michel [correspondant au 29 septembre du calendrier] se place quatre jours avant la Saint-François [4 octobre], laquelle a lieu un "mois » avant la Toussaint et tombe le même jour de la semaine que cette dernière. La Saint-André [30 novembre] se place un " mois " après la fête des Morts. La Saint-François et la Saint-André délimitent le " temps des morts » [miuhca tonalli]. (Cuacuila 1970)

Comme on le constate, l'information ne couvre pas toute l'année rituelle, mais seulement une fraction, qui concerne des rites obligatoires dans toutes les familles, la fête des Morts [miuhca iluitl], aussi nommée Toussaint («Todosantos », de l'espagnol Todos Santos). J'ai cru longtemps à tort que le caractère partiel de l'information venait de données incomplètes. Pourtant, j'avais l'explication dans mes notes : pour les Nahuas, la célébration de la fête des Morts, contrairement à d'autres fêtes religieuses, ne souffre pas d'être déplacée d'un seul jour. Cette croyance se retrouve dans tous les groupes indiens et dans les couches populaires de la région. Les gens se transmettent de petits contes qui ont tous pour morale la nécessité, à peine de punition surnaturelle mortelle, de fêter dignement les Morts et, de plus, à la date et au moment dits. Il existe donc une raison religieuse d'être particulièrement précis pour déterminer les dates de la Toussaint et du temps des Morts.

Voici une de ces histoires, contée en espagnol par une femme originaire de Cuacuila :

Un homme dit à sa femme : « Nous n'allons pas dresser la table des Morts cette année. Après tout, rien n'est moins sûr. Ce n'est pas vrai que les Morts viennent prendre les offrandes ». La femme se mit à pleurer : « Ce n'est pas bien de dire cela. Mon père et ma mère sont morts : nous devons faire les offrandes, si petites soient-elles ». Mais l'homme ne lui donna pas d'argent pour acheter le nécessaire. Aussi, quand la Toussaint arriva, la femme dut se contenter de déposer sur la table des pousses comestibles cueillies dans les champs [considérées comme un aliment de misère], quelques galettes de maïs et, comme elle n'avait pas de cierges, elle mit des torches de pin. L'homme indifférent s'en fut aux champs, comme à l'ordinaire. Il monta sur un arbre et commença à couper les branches. Soudain, il tomba et se tua. Il vit alors les Morts qui revenaient du village, 
chargés de bonnes choses à manger, et ses Morts à lui, avec les pousses et les galettes. Ces derniers lui reprochèrent amèrement ce piètre repas. L'homme se releva, vivant, ressuscité. Il retourna chez lui et raconta à tous les voisins ce qu'il avait vu : les Morts viennent vraiment à la Toussaint prendre la nourriture et les cadeaux sur les tables. Il dit alors à sa femme de faire des offrandes convenablement. Mais le temps des Morts était passé. Son repentir venait trop tard. L'homme, en effet, mourut dans l'année. (Chamoux 1997)

Revenons au mode de calcul et examinons de près le mode de repérage des fêtes de septembre à novembre en employant deux manières différentes de compter coexistant dans notre culture quotidienne. Pour suivre avec plus de facilité, on peut se reporter à l'extrait de calendrier reproduit ci-après (Tableau 1).

\begin{tabular}{|c|c|c|c|c|c|c|c|c|}
\hline \multicolumn{3}{|c|}{ Septembre } & \multicolumn{3}{|c|}{ Octobre } & \multicolumn{3}{|c|}{ Novembre } \\
\hline 1 & Mar & & 1 & Jeu & & 1 & Dim & Toussaint \\
\hline 2 & $\mathrm{Mer}$ & & 2 & Ven & & 2 & Lun & Defunts \\
\hline 3 & Jeu & & 3 & Sam & & 3 & Mar & \\
\hline 4 & Ven & & 4 & Dim & Saint-François & 4 & Mer & \\
\hline 5 & Sam & & 5 & Lun & & 5 & Jeu & \\
\hline 6 & Dim & & 6 & Mar & & 6 & Ven & \\
\hline 7 & Lun & & 7 & Mer & & 7 & Sam & \\
\hline 8 & Mar & & 8 & Jeu & & 8 & Dim & \\
\hline 9 & Mer & & 9 & Ven & & 9 & Lun & \\
\hline 10 & Jeu & & 10 & Sam & & 10 & Mar & \\
\hline 11 & Ven & & 11 & Dim & & 11 & Mer & \\
\hline 12 & Sam & & 12 & Lun & & 12 & Jeu & \\
\hline 13 & Dim & & 13 & Mar & & 13 & Ven & \\
\hline 14 & Lun & & 14 & Mer & & 14 & Sam & \\
\hline 15 & Mar & & 15 & Jeu & & 15 & Dim & \\
\hline 16 & Mer & & 16 & Ven & & 16 & Lun & \\
\hline 17 & Jeu & & 17 & Sam & & 17 & Mar & \\
\hline 18 & Ven & & 18 & Dim & & 18 & Mer & \\
\hline 19 & Sam & & 19 & Lun & & 19 & Jeu & \\
\hline 20 & Dim & & 20 & Mar & & 20 & Ven & \\
\hline 21 & Lun & & 21 & Mer & & 21 & Sam & \\
\hline 22 & Mar & & 22 & Jeu & & 22 & Dim & \\
\hline 23 & Mer & & 23 & Ven & & 23 & Lun & \\
\hline 24 & Jeu & & 24 & Sam & & 24 & Mar & \\
\hline 25 & Ven & & 25 & Dim & & 25 & Mer & \\
\hline 26 & Sam & & 26 & Lun & & 26 & Jeu & \\
\hline 27 & Dim & & 27 & Mar & & 27 & Ven & \\
\hline 28 & Lun & & 28 & Mer & & 28 & Sam & \\
\hline 29 & Mar & Saint-Michel & 29 & Jeu & & 29 & Dim & \\
\hline 30 & Mer & & 30 & Ven & & 30 & Lun & Saint-Andre \\
\hline & & & 31 & Sam & & & & \\
\hline
\end{tabular}

TABLEAU 1. - Calendrier de septembre à novembre (année de référence 1970) 


\section{Premier mode de calcul}

De nos jours, nous avons l'habitude de compter 1 jour à chaque fois que se termine un cycle diurne. Tout se passe comme si le début du compte partait d'un zéro initial (début du premier jour) et marquait un à la fin du premier cycle.

Cette manière de compter les jours semble avoir été celle des anciens Mexicains. On peut le déduire du lexique nahuatl de l'époque classique, d'après un dictionnaire fameux du Xvi ${ }^{\mathrm{e}}$ siècle (Molina $\left.1571 ; 1970\right)^{4}$. Doù il ressort d'ailleurs que l'idée du zéro, dont on parle beaucoup au sujet des anciens Mayas, n'était pas l'exclusivité de ces derniers dans l'aire méso-américaine. C'était sans doute une notion banale, au moins implicite, puisque la langue aztèque de tous les jours portait la trace, sinon d'un terme pour dire zéro, du moins d'une place pour le zéro.

Ce n'était pas la manière de compter le temps des Espagnols à l'époque de la Conquête et aux débuts de la colonisation. Ce que l'on constate dans le tableau ci-dessous - construit à partir du dictionnaire de Molina - en comparant la première colonne et sa traduction avec la troisième colonne et sa traduction (ibid., partie espagnol-nahuatl, p. 90 et, partie nahuatl-espagnol, p. 36) :

\begin{tabular}{|c|c|c|c|}
\hline Vieil espagnol & Traduction & Nahuall classique & Traduction \\
\hline Oy & Aujourdhui & Axcan & Aujourdhui \\
\hline Oy a tres días, & D'aujourd hui à trois jours & Yeouiptla & Après-demain \\
\hline Antier & Avant-hier & & Avant-hier \\
\hline Oy a cuatro días & D'aujourd'hui à quatre jours & $\begin{array}{l}\text { Yeoueyopan } \\
\text { (Yeoyeyupa) }\end{array}$ & Dans trois jours \\
\hline Antantier & & & Avant-avant-hier \\
\hline Oy a cinco dias & D'aujourd'hui à cing jours & Yeouenauhyopan & Dans quatre jours \\
\hline
\end{tabular}

TABleau 2. - Le compte des jours au Xvie siècle

Quand il part du nahuatl, Molina traduit littéralement cette langue et doit préciser la manière de compter : Yeoyeyupa traduit en vieil espagnol « tres días ha fin oy » [il y a trois jours à partir de la fin d'aujourd'hui] (ibid., partie nahuatlespagnol, p. 36).

Si nous faisons le compte à la façon contemporaine, qui est aussi la manière aztèque, le repérage des quatre fêtes dont nous avons parlé donne l'impression d'être plutôt imprécis :

- entre le 29 septembre et le 4 octobre, nous comptons cinq jours et non pas quatre, comme le dit l'informateur ;

- entre la Saint-François et la Toussaint [ $1^{\mathrm{er}}$ novembre], nous trouvons vingthuit jours, ce qui pourrait correspondre à un « mois » lunaire ; 
- entre la Toussaint [ $1^{\mathrm{er}}$ novembre] et la Saint-André le 30 novembre nous obtenons vingt-neuf jours, ce qui ne correspond ni à un « mois » lunaire ni à un " mois » grégorien à cette époque de l'année.

Même si, au lieu de prendre comme repère la Toussaint, on prend la fête des Morts officielle du calendrier rituel chrétien le 2 novembre, l'absence de mesure unifiée du « mois » reste la même (on a alors vingt-neuf jours avant et vingt-huit jours après, au lieu du contraire).

\section{Second mode de calcul}

À la façon ancienne commune en Europe au $\mathrm{XVI}^{\mathrm{e}}$ siècle on comptait pour un le premier jour dès son début. C'est la façon dont est initialisé le calendrier grégorien qui, comme chacun sait, n'a pas d'année zéro. Cela survit aussi dans les expressions « dans huit jours " pour dire une semaine (de sept jours comptés de façon moderne) et " dans quinze jours » pour dire deux semaines (de quatorze jours modernes). Dans cette hypothèse, le décompte indien des jours ne montre pas plus d'exactitude :

- entre le 29 septembre et le 4 octobre, on obtient six jours et non pas quatre, comme le dit l'informateur ;

- entre la Saint-François [4 octobre] et le $1^{\mathrm{er}}$ novembre, vingt-neuf jours ;

- entre la Toussaint [ $1^{\mathrm{er}}$ novembre] et la Saint-André, le 30 novembre, trente jours, seul nombre qui corresponde au « mois » grégorien de novembre.

Aucun de ces deux modes de calcul ne donne donc de résultats satisfaisants. Faut-il croire pour autant que notre informateur se satisfait d'une approximation assez grossière, à un ou deux jours près ? Adopter cette conclusion conduit à réintroduire la notion de " temps flottant ». Nous avons donc cherché s'il n'existait pas, sous-jacent, un autre mode de calcul, un autre mode de représentation du comput, et nous avons trouvé un modèle possible.

\section{LA PRÉCISION DU CALCUL SOUS-JACENT}

Dans nos habitudes mentales, nous concevons le temps balisé par le calendrier de façon arithmétique. Nous l'imaginons comme une droite continue, divisée en unités de base égales (jours) qu'on groupe suivant des césures régulières pour former des unités plus grandes, des semaines et des mois. $\mathrm{Si}$, au lieu de cela, on voit le temps de façon topologique, c'est-à-dire comme une série d'ensembles de jours ayant certains rapports entre eux et sur lesquels on peut faire des opérations, alors le temps des paysans nahuas devient beaucoup moins « flottant » qu'il n'y paraît.

Quelques précisions s'imposent. On dira plutôt « cycle diurne » que « jour » pour éviter des confusions; en effet, chez les Nahuas, le début d'un cycle diurne 
religieux est différent de celui du calendrier grégorien. Selon le calendrier grégorien, minuit est le début d'un nouveau jour, le moment du changement de date. Selon les pratiques rituelles indiennes, le début du cycle diurne est vraisemblablement midi, le midi qui précède le changement de date grégorien (ainsi, le début de la Toussaint est le 31 octobre à midi, et non au changement de date, à minuit).

Dans notre culture, nous disons que chaque jour porte le nom d'un saint, mais nous savons qu'un Indien de la Méso-Amérique ancienne disait plutôt que des dieux portaient des jours (et du temps). Quelle que soit la formulation, l'idée n'est pas très différente. Les saints et les fêtes - Saint-Michel, Saint-François, SaintAndré, Toussaint et fête des Morts - peuvent être considérés comme portant des jours ou portés par ces derniers. Ce qui est important ici, c'est de les considérer comme bornant des ensembles.

Sommairement, on dit que si l'ensemble contient ses bornes, il est fermé. S'il ne contient pas au moins une borne, il est ouvert. Ces idées simples organisent parfaitement les données recueillies. En cherchant à glisser les jours bornes dans l'un ou l'autre ensemble de façon à respecter les nombres de jours indiqués par les informateurs, il apparaît des régularités. Elles sont résumées dans le tableau qui suit, où l'on dégage cinq ensembles nommés $\mathrm{A}, \mathrm{B}, \mathrm{C}, \mathrm{D}, \mathrm{E}$, les uns fermés, les autres ouverts.

\begin{tabular}{|c|c|c|c|}
\hline Dates & Fêtes & appartient à & \\
\hline $\begin{array}{l}\text { date inconnue } \\
28 / 29 \text { sept. }\end{array}$ & $\begin{array}{l}\text { fête inconnue } \\
\text { Saint-Michel }\end{array}$ & $\begin{array}{l}\text { ensemble A - borne inconnue } \\
\text { ou inexistante } \\
\text { ensemble A - borne }\end{array}$ & appartient à l'ensemble $\mathrm{A}$ \\
\hline $\begin{array}{l}29 / 30 \text { sept. } \\
30 \mathrm{sept} / 1^{\text {"t }} \mathrm{oct} . \\
1^{\text {ct/ } / 2 \mathrm{oct} .} \\
2 / 3 \text { oct. }\end{array}$ & $\begin{array}{l}\text { jour } n^{\circ} 1 \\
\text { jour } n^{\circ} 2 \\
\text { jour } n^{\circ} 3 \\
\text { jour } n^{\circ} 4 \\
\end{array}$ & $\begin{array}{l}\text { ensemble B } \\
\text { ensemble B } \\
\text { ensemble B } \\
\text { ensemble B } \\
\end{array}$ & \\
\hline $\begin{array}{l}3 / 4 \text { oct. } \\
\text { octobre } \\
30 / 31 \text { oct. }\end{array}$ & $\begin{array}{l}\text { Saint-François, } n^{\circ} 1 \\
\text { jours } n^{\circ} 2 \text { à } 27 \\
\text { jour } n^{\circ} 28 \\
\end{array}$ & $\begin{array}{l}\text { ensemble C - borne } \\
\text { ensemble C } \\
\text { ensemble C - borne }\end{array}$ & $\begin{array}{l}\text { appartient à l'ensemble C } \\
\text { appartient à l'ensemble C }\end{array}$ \\
\hline $\begin{array}{l}31 \mathrm{oct} / 1^{e t} \text { nov. } \\
1^{\mathrm{t} /} / 2 \mathrm{nov} .\end{array}$ & $\begin{array}{l}\text { tlaxochitepeualistli } \\
\text { huehueyin }\end{array}$ & $\begin{array}{l}\text { ensemble D } \\
\text { ensemble D }\end{array}$ & \\
\hline $\begin{array}{l}2 / 3 \text { nov. } \\
\text { novembre } \\
29 / 30 \text { nov. }\end{array}$ & $\begin{array}{l}\text { tlacualxelolistlli } \\
\text { jours } n^{\circ} 2 \text { à } 27 \\
\text { Saint-André, } n^{\circ} 28\end{array}$ & $\begin{array}{l}\text { ensemble } \mathbf{E} \text { - borne } \\
\text { ensemble E } \\
\text { ensemble } \mathrm{E} \text { - borne }\end{array}$ & $\begin{array}{l}\text { appartient à l'ensemble E } \\
\text { appartient à l'ensemble E }\end{array}$ \\
\hline
\end{tabular}

Tableau 3. - Dates de fêtes et découpage en ensemble

Saint-Michel est par définition une borne. Nous le faisons appartenir à un ensemble fermé, dont nous ignorons l'autre borne, faute de données ethnographiques sur ce point : nommons-le A. Saint-François est une autre borne, qui, pour répondre au décompte de jours recueilli, doit être placé dans un ensemble $C$, 
fermé. Entre les deux, il y a un ensemble, nommé ici B, qui n’a pas de bornes et est donc ouvert, et qui comprend quatre jours, exactement comme les informateurs l'ont dit (la Saint-Michel, c'est quatre jours avant la Saint-François). L'ensemble suivant, C, qui est fermé, commence au jour borne de la Saint-François. Sur le plan des croyances, il appartient au « temps des Morts » (les Morts se mettent en route), dans l'Avant de la Toussaint. Cet ensemble dure jusqu'au 31 octobre à midi. Il n'y a pas de nom de saint employé pour marquer ce jour, mais en le prenant comme borne incluse, l'ensemble $\mathrm{C}$ a vingt-huit jours, conformément au compte indigène recueilli.

Le 31 octobre, à partir de midi, commence la préparation directe de la fête des Morts. Une intense activité culinaire et de dressage de l'autel des Morts se déploie. Cette période est nommée tlaxochitepeualistli, l'éparpillement des fleurs, qui est le moment d'arrivée des « petits » morts, des enfants décédés censés venir se nourrir pendant la nuit du 31 octobre au $1^{\text {er }}$ novembre. Dans l'après-midi du $1^{\text {er }}$ novembre, on achève de préparer les offrandes pour huehueyin, moment de l'arrivée des morts adultes, dans la nuit du $1^{\text {er }}$ au 2 novembre. Ces deux cycles diurnes, du 31 octobre à midi au 2 novembre à midi, constituent le point culminant du rituel et de la fête des Morts proprement dite, et forment l'ensemble D (les Morts sont arrivés). On ne peut dire s'il est ouvert ou fermé car il ne comporte que deux cycles. Il constitue en tout cas le repère central à partir duquel les Nahuas pensent le temps de cette partie de l'année.

Le 2 novembre à midi commence l'Après du " temps des Morts » : les Morts ayant reçu leurs offrandes font le voyage du retour. C'est alors le tlacualxelolistli, le partage des mets, consacré par la coutume à des visites aux parrains et à des dons de nourriture entre marraines et filleules. Ce cycle diurne du $2 / 3$ novembre peut être considéré comme une borne de l'ensemble suivant, ou ensemble $\mathrm{E}$, qui finit le 29/30 novembre, à la Saint-André. Si l'on compte le 2/3 novembre comme une borne ainsi que la Saint-André (29/30 novembre, jour très important dans les rites indigènes), l'ensemble dure vingt-huit jours au total.

Cette manière d'organiser le temps en termes d'ensembles rend bien compte des informations de terrain en ce qui concerne les nombres de jours. Abordé de cette façon, le calcul des dates de fêtes n'a plus rien d'approximatif ni de «flottant ».

\begin{tabular}{|l|l|l|l|}
\hline Ensemble & Dates & Type & Nombre de cycles \\
\hline ensemble A & du ? au28/29 sept. à midi & fermé & x cycles diurnes \\
ensemble B & du29/30 sept au2/3 oct. & ouvert & 4 cycles diurnes \\
ensemble C & du3/4 au30/31 oct. à midi & fermé & 28 cycles diurnes \\
ensemble D & du31 oct./1" nov au2 nov. à midi & indéterminé & 2 cycles diurnes \\
ensemble E & du2/3 au 29/30 nov. & fermé & 28 cycles diurnes \\
\hline
\end{tabular}

TABLEAU 4. - Succession des ensembles 
HyPOTHÈSES SUR LES PROCÉDURES DE CALCUL ET LES REPRÉSENTATIONS MENTALES

D'après ces résultats, on peut formuler quelques hypothèses sur les procédures de calcul et les représentations mentales des Nahuas concernant le calendrier religieux.

\section{Hypothèse du chapelet temporel}

Le tableau 4, bien qu'il traite de données partielles, permet de se demander avec quelque raison s'il n'y a pas un principe d'alternance entre un ensemble fermé et un ensemble ouvert dans le système de division du temps : A serait fermé, $\mathrm{B}$ ouvert, $\mathrm{C}$ fermé, D indéterminé (ou ouvert ?), E fermé. Nous aurions là une régularité. C'est à l'ethnographie ou à l'étude des sources historiques de répondre en apportant si possible de nouvelles données. De façon analogique, on pourrait dire que le temps des fêtes religieuses peut être représenté comme un collier ou un chapelet, ou encore une cordelette, où alternent des perles, grains ou nœuds (ensembles fermés) et des intervalles (ensembles ouverts). Le temps se compte sur le chapelet, grain après grain, de la même manière qu'un ordinateur parcourt un algorithme. Cette procédure est compatible aussi bien avec un programme qui fait une boucle (cycle temporel) qu'avec un chemin linéaire (flèche du temps).
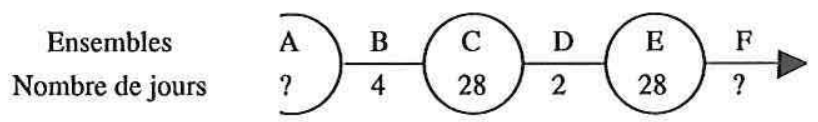

SCHÉmA 1. - Le chapelet temporel

\section{Hypothèse de la pliure du temps}

On ne peut par ailleurs manquer de remarquer la symétrie en miroir entre l'Avant et l'Après du temps des Morts : vingt-huit jours pour chaque période. La fête des Morts centrale comprend deux cycles diurnes et peut aussi se diviser en deux. 


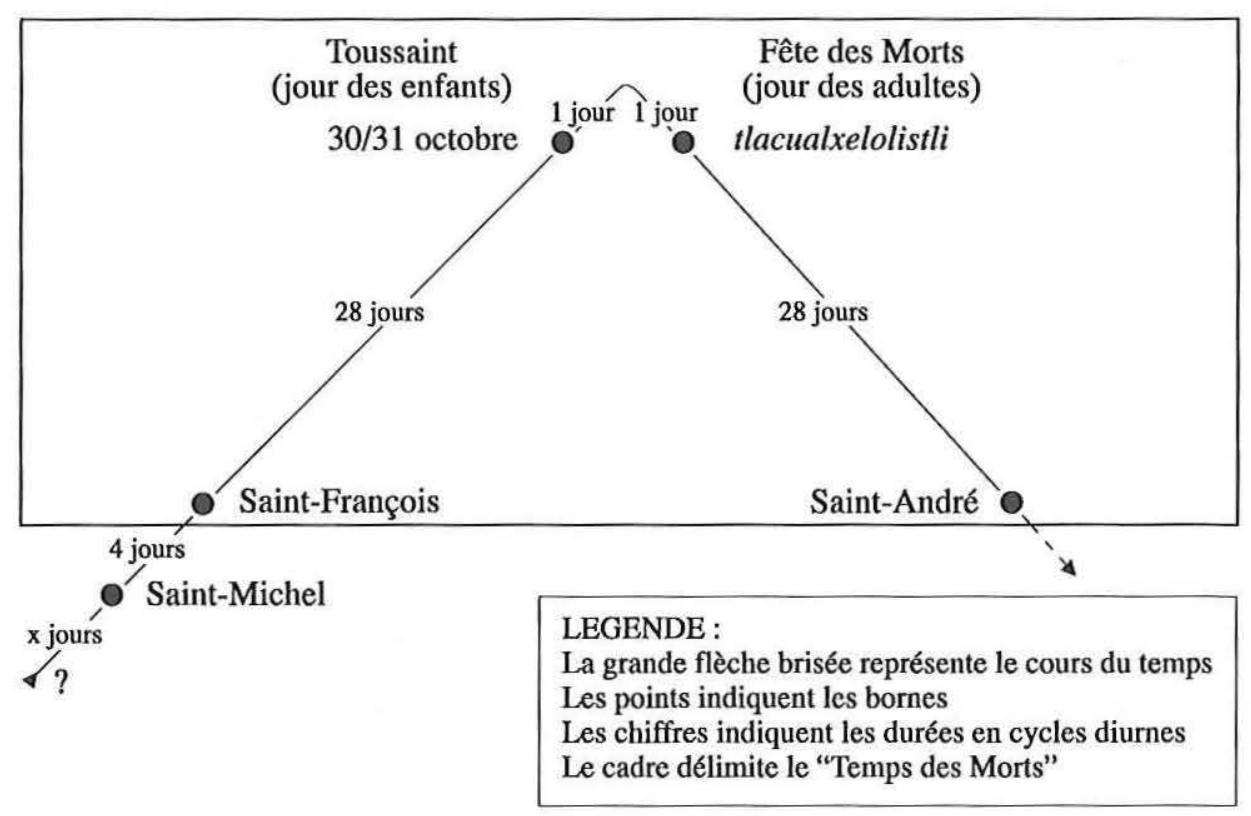

SCHÉMA 2. - La pliure temporelle

L'usage de ce genre de symétrie dans la délimitation de périodes à signification religieuse semble commune en Méso-Amérique. López Austin signalait récemment dans une conférence donnée à Paris que, chez les Tzotzils contemporains (groupe maya), on pensait qu'un mort connaissait dans l'au-delà un temps de purification égal en durée au temps qu'il avait vécu sur terre. Tout se passe comme si le temps se décomptait à partir d'événements importants ou connus de tous (la Toussaint, le décès, un rite...) qui servent de repères centraux et permettent de déterminer un Avant donnant par symétrie la mesure de la durée d'un Après. Le système semble établir une période étalon dont la durée est mesurée en jours et qui conduit jusqu'à un événement central. Le résultat de ce compte sert à déterminer le moment de l'événement final qui clôture la période rituelle.

On retrouve un principe semblable dans de nombreuses langues pour positionner couramment un événement récent. En français, nous avons : aujourd'hui (événement central), demain, hier, après-demain, avant-hier ; en espagnol contemporain : hoy (événement central), mañana, ayer, pasado mañana, anteayer. En nahuatl, axan (événement central: aujourd'hui), mostla (demain), ialua (hier), uiptla qui veut dire « après-demain » et également « avant-hier ». 
On a souvent dit que les populations méso-américaines ne distinguaient pas le passé du futur, en s'appuyant sur le fait que uiptla signifie aussi bien « aprèsdemain » que « avant-hier ». Cependant, contrairement à cette thèse répandue, j'ai tendance à penser qu'il ne s'agit pas de confusion, ni de bouclage circulaire, mais de mesure. Ce procédé est un mode de calcul et de quantification et non la manifestation d'une conception du temps cyclique « archaïque » où le passé et l'avenir se confondraient. Il y a superposition, et non pas fusion, l'Avant constituant un étalon de mesure et l'Après étant la durée mesurée. L'unicité du terme indique que la distance qui sépare de l'événement est la même, avant ou après, et non pas qu'il y a retour au point de départ.

La pliure peut également se représenter graphiquement sur le modèle ondulatoire. Plus fondamentalement, et bien au-delà du cas des Nahuas, la pliure constitue une des hypothèses fortes de procédure mentale universelle, avancée en mathématique, en linguistique et en sémantique. La pliure est un processus dynamique et irréversible (comme le Temps), qui détermine un Avant et un Après [-événement]. Le retour en arrière à l'identique (identité mathématique, $1=1$ ) est impossible, mais il peut y avoir similitude entre l'Avant et l'Après (ressemblance de formes, de propriétés, etc.). La pliure engendre le " cycle » (qui n'est pas synonyme d'éternel retour) et la dualité (1 devient 2) (Pottier 2001, pp. 10-11, s'appuyant sur la théorie des catastrophes de Thom).

\section{Le comput lunaire}

On aura remarqué, bien sûr, la période de vingt-huit jours qui évoque les phases de la lune. Les Nahuas nomment cette durée metztli, ce qui signifie " lune » et aussi « mois ». Il est connu que les Indiens totonaques voisins possèdent un calendrier lunaire et leurs ressemblances culturelles avec les Nahuas de cette région ont été remarquées depuis longtemps (Lombardo Toledano 19311932). Mais ce qui paraît plus important de souligner, c'est que les repères des Nahuas ne font pas intervenir d'autres éléments de leur environnement que le cycle diurne, base de tous les calendriers. Le système de repérage tel qu'il m'a été transmis par les informateurs ne fait pas référence directe à la lune. Il ne s'appuie pas sur l'observation des phases de notre satellite naturel. Il relève principalement d'un mode de calcul. La vérification de l'exactitude du compte des jours repose sur le retour du nom du jour de la semaine, qui est la semaine de sept jours du calendrier grégorien (" la Saint-François a lieu un "mois" avant la Toussaint et tombe le même jour de la semaine que cette dernière », dit l'informateur). Pour identifier le jour de la semaine, la coordination des activités humaines locales est suffisante car elle envoie en permanence des signaux de repérage (le samedi est jour de marché, le dimanche est jour de corvée communale, etc.). On ne peut exclure, bien sûr; que les Nahuas jettent parfois un coup d'œil aux phases de la 
lune pour voir où en est le compte des vingt-huit jours, mais je ne l'ai jamais vu faire ni entendu évoquer.

La procédure attestée jusqu'à plus ample information est l'usage du concept d'une période de vingt-huit jours (metztli, «lunaison », « mois »). Il se trouve qu'elle est divisible par quatre, ce qui détermine les semaines $(28 / 4=7)$. Les Nahuas se repèrent alors par les correspondances avec les noms de jours de la semaine de sept jours du calendrier grégorien, lesquels renvoient aux activités des hommes, et aussi à celles des dieux.

\section{Le groupement de nombre marqué}

Le nombre quatre, quant à lui, est un nombre des plus marqués dans les cultures méso-américaines anciennes et contemporaines : son usage constitue une des survivances précolombiennes. Dans la région étudiée, nous le voyons apparaître abondamment comme un groupement standard dans de nombreux domaines: dans les mythes, dans les rites, dans les contes, dans les manières ordinaires de parler des Nahuas d'aujourd'hui. C'était aussi un nombre clé à l'époque précolombienne - par exemple dans le mythe des quatre Soleils ou ères, débutant tous dans des années au nom commençant par quatre. Ce groupement de nombre est utilisé de nos jours dans le repérage de dates. Ce n'est pas le seul nombre marqué survivant dans les énoncés concernant le temps : signalons le chiffre vingt, qu'on trouve dans les expressions ordinaires (" Marie est restée vingt jours ») ou dans les prescriptions rituelles (abstinence de vingt jours). Mais ce nombre vingt n'est pas apparu dans les calculs de date recueillis et analysés ici.

Dans une ancienne étude, consacrée à la formalisation des plans de campements et de villages amérindiens et indonésiens, Claude Lévi-Strauss (1958) montrait qu'il ne s'agissait pas de coutumes arbitraires et curieuses, mais de transcriptions, dans l'espace habité, de manières de catégoriser le monde naturel et social, manières dont on peut retrouver les principes communs et rationnels. Dans les lignes qui précèdent, on a pu faire émerger un ordre et une certaine forme de précision au-delà d'une impression initiale de confusion et de flottement. On voit ainsi comment des paysans, pour la plupart analphabètes à l'époque de l'enquête, ont construit des procédures de calcul pour faire communiquer l'univers temporel rural, local et indien avec celui des autorités centrales diseuses de temps, l'Église et l'État. Ils ont converti le calendrier comme on convertit mentalement des monnaies étrangères, avec les outils numériques tirés de leurs schèmes culturels. Mais il y a autre chose que la mise en évidence d'un ordre sous-jacent. Par l'élaboration rigoureuse des données, des hypothèses ont pu être avancées sur des procédures de représentation du temps qui ne renvoient plus seulement aux poncifs qui polarisent classiquement les débats, en opposant un temps linéaire, moderne et raisonné, à un temps cyclique, archaïque et religieux. Il semble qu'il y ait d'autres modèles possibles pour concevoir et mesurer le temps. La pertinence 
de ceux évoqués ici - le chapelet, la pliure, les groupements de nombres - pourrait être soumise à l'épreuve de l'abondante documentation sur l'aire mésoaméricaine que livrent l'archéologie, l'ethnohistoire, la linguistique et l'anthropologie. On ajoutera encore que la tradition de l'école française de sociologie rapportant in fine la scansion du temps des populations " archaïques » aux activités économiques, religieuses et sociales montre une limite. Certes, les activités constituent un domaine de repères temporels disponibles et facilement observables, mais cela ne doit pas masquer qu'elles coexistent avec d'autres domaines de repères, y compris ceux résultant du savoir-faire de calcul mental. Ces divers domaines qui entrent en jeu dans la quantification du temps sont imparfaitement répertoriés et les procédures pour les mettre en correspondance sont encore largement à découvrir *

* Manuscrit reçu en novembre 2002, accepté pour publication en février 2003.

\section{NOTES}

1. Des traces ont cependant été retrouvées dans le monde maya, au Guatemala.

2. Nous devons à la rareté d'instruments de marquage horaire au $\mathrm{XvI}^{\mathrm{e}}$ siècle un des plus célèbres dialogues de tragédie : Roméo et Juliette se demandant s'ils entendent le rossignol ou l'alouette.

3. Les phénomènes évoqués ne sont plus visibles aujourd'hui. L'école et les avancées de la modernité ont répandu, dans les nouvelles générations, d'autres manières de se repérer dans le calendrier. Mais la trace des anciennes procédures vaut d'être conservée.

4. On appelle "nahuatl classique" la langue qui était parlée au Xvi ${ }^{e}$ siècle dans la région de Mexico.

\section{RÉFÉRENCES CITÉES}

BENSA A.

1997

«Images et usages du temps », Terrains. Vivre le temps, 29, pp. 5-18.

BERNot L. et R. BLANCARD

$1953 \quad$ Nouville : un village français, Institut d'ethnologie, Paris, coll. « Travaux et mémoires de l'Institut d'ethnologie » 57.

BRUNER J. S.

1977

A Study of Thinking, Textbook, New York [1956].

Chamoux M.-N.

1997

« La cuisine de la Toussaint chez les Aztèques de la Sierra de Puebla (Mexique) », L'Internationale de l'imaginaire, n.s. 7, pp. 85-99.

DURKHEIM E.

1968

Les Formes élémentaires de la vie religieuse: le système totémique en Australie, PUF, Paris [1912]. 
Evans-Pritchard E. E.

1968 Les Nuer. Description des modes de vie et des institutions politiques d'un peuple nilote, Gallimard, Paris.

FebVRe L.

1968 Le Problème de l'incroyance au xvi siècle. La religion de Rabelais, Albin Michel, Paris.

Mauss M.

1969 Euvres 1, Éditions de Minuit, Paris [1906, 1909].

IZARD M.

1990 "Temps » in P. Bonte et M. Izard, éds, Dictionnaire de l'ethnologie et de l'anthropologie, PUF, Paris, pp. 702-703.

Lévi-Strauss C.

1958

« Les organisations dualistes existent-elles ? » in C. Lévi-Strauss, Anthropologie structurale, Plon, Paris, pp. 147-180.

Lombardo Toledano V.

1931-1932 Geografia de las lenguas de la Sierra de Puebla, vol, 3, n 13, UNAM, México.

Molina Fray Alonso de

1970 Vocabulario en lengua castellana y mexicana y mexicana y castellana, edición fac-similé, Editorial Porrúa, México [1571].

PotTier B.

2001 Représentations mentales et catégorisations linguistiques, Peeters, Louvain-Paris. 